\title{
Neuroendocrine neoplasms of the pancreas: diagnosis and pitfalls
}

\author{
Björn Konukiewitz ${ }^{1}$ - Moritz Jesinghaus ${ }^{2} \cdot$ Atsuko Kasajima $^{3} \cdot$ Günter Klöppel $^{3}$
}

Received: 6 September 2021 / Revised: 6 September 2021 / Accepted: 21 September 2021 / Published online: 13 October 2021

(c) The Author(s) 2021

\begin{abstract}
Common to neuroendocrine neoplasms of the pancreas is their expression of synaptophysin, chromogranin A, and/or INSM1. They differ, however, in their histological differentiation and molecular profile. Three groups can be distinguished: welldifferentiated neuroendocrine neoplasms (neuroendocrine tumors), poorly differentiated neuroendocrine neoplasms (neuroendocrine carcinomas), and mixed neuroendocrine-non-neuroendocrine neoplasms. However, the expression of synaptophysin and, to a lesser extent, also chromogranin A is not restricted to the neuroendocrine neoplasms, but may also be in a subset of non-neuroendocrine epithelial and non-epithelial neoplasms. This review provides the essential criteria for the diagnosis of pancreatic neuroendocrine neoplasms including diagnostic clues for the distinction of high-grade neuroendocrine tumors from neuroendocrine carcinomas and an algorithm avoiding diagnostic pitfalls in the delineation of non-neuroendocrine neoplasms with neuroendocrine features from pancreatic neuroendocrine neoplasms.
\end{abstract}

Keywords Pancreatic neuroendocrine neoplasms $\cdot$ Diagnosis $\cdot$ Histology $\cdot$ Immunohistology $\cdot$ Pitfalls

\section{Introduction}

Diagnosis and pitfall are like two sides of the same coin. The better the diagnostic criteria, the less the number of diagnostic pitfalls. However, careful processing of diagnostic failures has helped in many cases to improve diagnostic criteria. In this article on pancreatic neuroendocrine neoplasms (PanNENs), we will therefore focus on both, presenting the most important diagnostic criteria and providing clues to avoid main pitfalls.

PanNENs belong to the tumors that bear the generic name "neuroendocrine neoplasms." This name is used as a collective term for two tumor families that share the expression of neuroendocrine markers, such as synaptophysin and chromogranin A, but differ distinctly in their morphological and molecular profiles [1, 2]. In the first group, growth and

Björn Konukiewitz

Bjoern.Konukiewitz@uksh.de

1 Institute of Pathology, Universitätsklinikum SchleswigHolstein, Campus Kiel, Christian-Albrechts-Universität zu Kiel, Arnold-Heller-Straße 3/14, 24105 Kiel, Germany

2 Institute of Pathology, Universitätsklinikum Marburg, Baldingerstraße, 35043 Marburg, Germany

3 Institute of Pathology, Technische Universität München, Trogerstraße 18, 81675 Munich, Germany behavior is slower and individually more different than in the second group, where it is generally faster [3]. Both groups of NENs can arise almost anywhere in the body, even though they all show a strong preference for the gastroenteropancreatic system and the lung, with a varying and interesting site-specific distribution. For historical reasons, the world health organization (WHO) classifications of the NENs of the various organ systems do not follow a uniform terminology [4]. However, the WHO generally follows the principle of distinguishing between well and poorly differentiated NENs and the delimitation of mixed neoplasms [1, 2, 5-7].

The PanNENs play a pioneering role in the classification of NENs because they are frequent among the NENs, have a very varied morphology, and may show a multifaceted functionality [1]. Currently, they are represented in two WHO "blue books," the classifications of tumors of endocrine organs and the classification of digestive system tumors [1, 2]. This review aims to outline the morpho-genetic characteristics of pancreatic NENs and to provide a practical approach to daily routine diagnostics with highlighting of main diagnostic pitfalls and important NEN mimics. 


\section{Diagnostic features}

The 2017 and 2019 WHO classifications stratify the PanNENs into well-differentiated NENs (pancreatic neuroendocrine tumors, PanNETs) and poorly differentiated NENs (pancreatic neuroendocrine carcinomas, PanNECs) and presume that all PanNENs have a malignant potential, however, with a different probability to metastasize [1,2].

\section{PanNET}

The histological profile of PanNETs is characterized by an organoid growth pattern with an ordered arrangement of cells, a variable amount of fibrotic stroma, and only rare necrotic changes (Figs. 1 and 2). Although the organoid pattern is not uniform but very diverse, the histological diversity can probably be traced back to either a solid or trabecular architecture that can be subdivided due to the composition of the stroma and its vascularization into solidnested, solid-paraganglioma-like, solid-microglandular, and trabecular-reticulated, trabecular-gyriform, and trabecularcystic patterns [8-11]. Interestingly, some of these patterns seem to have a relationship to the functionality of the tumor cells, because they show a strong association with the expression of certain hormones (see below).

The cells of this organized tumor tissue mostly display an eosinophilic cytoplasm and mainly round uniform nuclei with hyperchromatic (pepper and salt) chromatin, small inconspicuous nucleoli, and a rather low mitotic rate. PanNETs with oncocytic, lipid-rich (clear), or hepatoid cells or with pleomorphic nuclei are rare [11]. PanNETs are
Fig. 1 Neuroendocrine tumors with solid (A) and trabecular (B) growth patterns
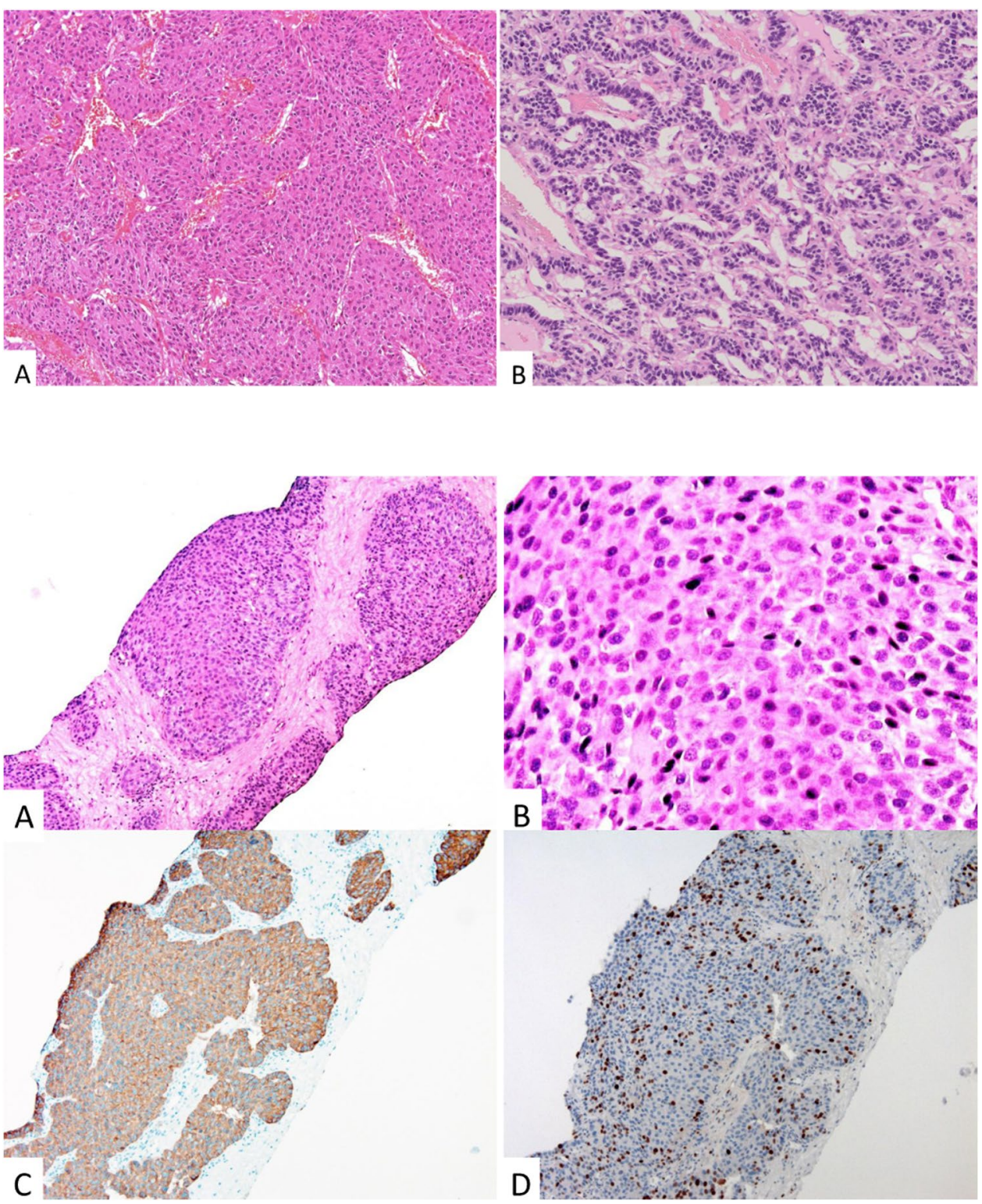

Fig. 2 Liver metastasis of a neuroendocrine tumor G3 with an organoid growth pattern (A) showing monomorphous and round nuclei (B) and expression of synaptophysin (C) and Ki67 (index 25\%) (D) 
usually well demarcated from the surrounding parenchyma, when they are small $(<1 \mathrm{~cm})$. When they are larger, they can widely infiltrate into the adjacent acinar tissue, thereby invading vessels and nerves and entrapping preexistent islets or single ducts. Rare PanNETs show a peculiar mixture of solid or trabecular cell clusters with small non-neoplastic ducts often embedded in the sclerotic stroma [1-3, 12,13].

The immunohistochemical profile of PanNETs that is essential to establish the diagnosis includes the expression of cytokeratin, synaptophysin, and chromogranin A and Ki67 (Fig. 2) [14]. In the case of a PanNET, G3 staining for p53 and RB1 is highly recommended to distinguish these tumors from PanNECs (Fig. 3). The staining of peptide hormones, of the somatostatin receptor 2A (SST2) or the site-specific transcription factor ISLET-1, is recommended where the diagnosis needs it to be complete $[4,8,15,16]$.

Labeling for cytokeratin proves the epithelial nature of a NEN in cases where a neuroectodermal tumor such as a paraganglioma must be excluded [10]. Diffuse and intense cytoplasmic expression of synaptophysin and chromogranin A and nuclear staining for insulinoma-associated 1 (INSM1) (Fig. 3) [17] reveals the tumor's neuroendocrine differentiation, the common denominator of NENs [18]. The labeling of the nuclei with Ki67 is the best way to accurately determine the proliferative activity of the tumor cells, and this method has largely replaced the counting of mitoses. The exact assessment of the proportion of Ki67-labeled cells as the basis for the calculation of the Ki67 index has emerged as indispensable for the prognostic and therapeutic stratification of PanNETs $[19,20]$. The stratification is based on a three-tired grading that separates G1, G2, and G3 PanNETs according to their Ki67 index (Table 1) [1, 2]. PanNETs G3, which represent a new category among NENs, have no defined upper mitotic or Ki67 rate limit; however, usually their mitotic rate and Ki67 index do not exceed 20/10 HPF and 50\% (Fig. 2), respectively. Most PanNETs G3 appear to develop from a low-grade NET, since they often manifest themselves as metastases in patients with a prior history of a G1 or G2 PanNET [21].

PanNETs produce peptide hormones which are orthotopic (insulin, glucagon, somatostatin, pancreatic polypeptide, and serotonin) or ectopic (gastrin, vasoactive intestinal polypeptide; VIP, adrenocorticotropin; ACTH; and others) to the pancreas and can be identified by specific antibodies. Approximately $30 \%$ of PanNETs are functioning, meaning that the peptide hormone which is produced and secreted also causes a hormonal syndrome. The functioning NETs of the pancreas include insulinoma, glucagonoma, gastrinoma, VIPoma, GRHoma, ACTHoma, or PanNET with

Table 1 Grading of pancreatic neuroendocrine neoplasms (adapted from (1))

\begin{tabular}{lllll}
\hline & Morphology & Grade & Ki67 index* & Mitosis** \\
\hline NET & Well differentiated & G1 & $<3 \%$ & $<2$ \\
& & G2 & $3-20 \%$ & $2-20$ \\
& & G3 & $>20 \%$ & $>20$ \\
NEC & Poorly differentiated & & $>20 \%$ & $>20$ \\
\hline
\end{tabular}

Abbreviations: NET neuroendocrine tumor, $N E C$ neuroendocrine carcinoma

*Counted in at least 500 cells in hot spot areas, **in $2 \mathrm{~mm}^{2}$
Fig. 3 Neuroendocrine carcinoma, large cell type: solid cell clusters with pleomorphic nuclei showing a prominent nucleolus (A), and an overexpression of p53 (B). Fine-needle aspiration cytology specimens with matching expression of synaptophysin (C) and INSM1 (D)
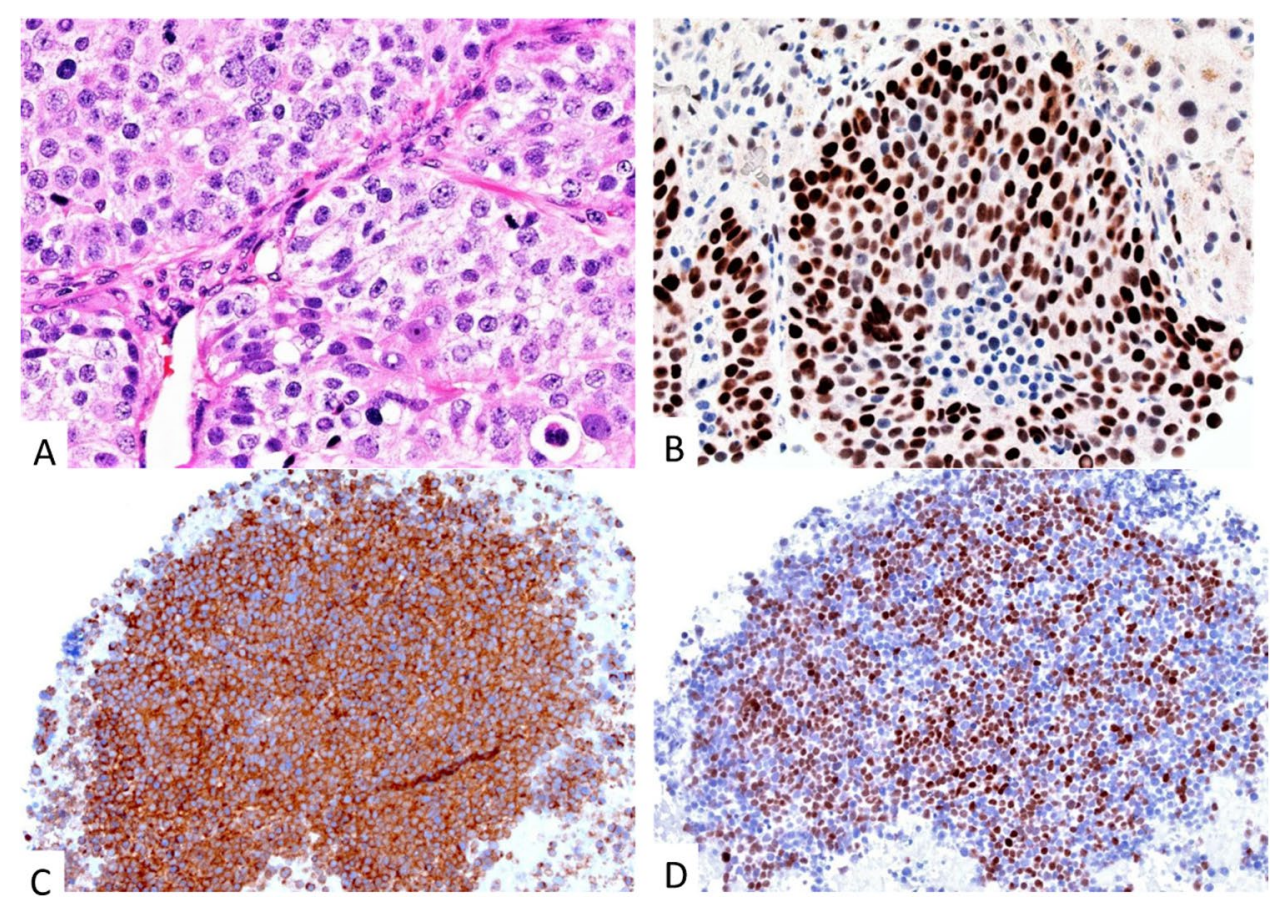
carcinoid syndrome and serotonin expression $[1,2]$. Whether PanNETs producing and secreting somatostatin can cause a somatostatin syndrome, as described in 1979, is currently under debate, since a study from 2008 was unable to find any syndrome in somatostatin-positive PanNETs or duodenal NETs, and the evidence given in the most recent study is inconclusive [22, 23]. Rarely, there are PanNETs with hypercalcemia, which may produce calcitonin, but the occurrence of hypercalcemia is not necessarily tied to calcitonin secretion $[2,24]$. The most frequent functioning PanNETs are insulinomas, which in $90 \%$ are small $(<2 \mathrm{~cm})$ and behave benignly [25]. All the other functioning PanNETs are rare and usually show a malignant behavior, especially the tumors with ACTH production and Cushing syndrome. All PanNETs that produce and also secrete a hormone, but are non-syndromic, fall into the category of non-functioning PanNETs and account for about $70 \%$ of all PanNETs. Forty percent of PanNETs are multihormonal and are generally found among the non-functioning tumors [2]. Interestingly, the rare (about 20\%) malignant insulinomas seem to start in the pancreas as non-functioning multihormonal tumors, with only single insulin cells, but become syndromic after large liver metastases have developed in which the number of insulin-secreting cells is sufficient to produce a hypoglycemic syndrome (GK, personal observation).

It is increasingly noticed that the production of some hormones is associated with certain histological patterns of the PanNETs. Thus, a solid-nested pattern with amyloid is usually associated with the expression of insulin and is found in insulinomas [1]. Tumors with a trabecular-reticulated and often cystic pattern express glucagon [9]. Tumors with a solid paraganglioma-like or solid-microglandular pattern with psammoma-bodies usually contain somatostatin-positive cells [10], and a trabecular sclerosing pattern of a tumor adjacent to the main duct commonly associates with serotonin positive cells [26]. In some multihormonal PanNETs with a clear separation of a solid from a trabecular pattern, each pattern may have its own hormone production.

Membranous SST2 expression on tumor cells is needed to visualize and treat the tumors with radioisotope-labeled somatostatin [16]. It can be detected in most PanNETs, except for insulinomas which express SST2 in only 50\% of the tumors and rather express GLP1R than SST2 [27]. If a primary tumor is SST2-positive, it can be assumed that later metastases are also positive and are therefore detectable in the follow-up. Very helpful for the localization of a primary in the pancreas (or duodenum) in case of a liver metastasis with unknown primary is the nuclear expression of the transcription factor ISLET-1 [4, 8, 28].

The molecular profile of PanNET is profoundly different from that of pancreatic ductal adenocarcinomas (PDAC). Key drivers of PanNETs are alterations in MENI and ATRX or DAXX [29-33], while abnormalities of KRAS, TP53, CDKN2A, and SMAD4 are the drivers in PDACs [34-36]. MEN1 is a tumor suppressor gene located on chromosome 11 [37] encoding for the protein Menin, which is an important factor for the regulation of chromatin remodeling [38-40] and seems to play a key role in tumor initiation as $M E N 1$ alterations are already detectable in pancreatic microtumors [41] and as germline mutation in the genetic syndrome multiple endocrine neoplasia type 1 (MEN1, see below). MEN1 is known to interact with genes of chromatin modifications, altered telomere length, DNA damage repair, and mTOR signaling, which are the four main genetic pathways involved in the development of pancreatic NETs [42]. ATRX and DAXX are also genes involved in chromatin remodeling with a high frequency of alterations (40\%) in PanNETs [29]. Inactivating mutations of $A T R X$ or $D A X X$ are associated with an alternative lengthening of telomeres (ALT), a telomerase-independent telomere maintenance mechanism [30]. ATRX/DAXX alterations seem to be late events in tumorigenesis as they are only detectable in large fully developed NET [31, 32] but not in microadenomas [41]. Furthermore, NET with $A T R X / D A X X$ mutations appear to be associated with a poor prognosis compared to $A T R X / D A X X$ wildtype tumors [31, 32, 42]. ATRX and DAXX are genes that are strongly involved in different epigenetic mechanisms regulating gene expressions per methylation pattern [43]. MEN1, $A T R X$, and DAXX alterations are associated with a pathological protein expression which is detectable by immunohistochemistry [29, 44]

The third relevant cluster of commonly mutated genes are alterations in genes belonging to the mTOR pathway, which are mutated in about $15 \%$ of the pancreatic NET [29, 42], mostly affecting PTEN, TSC1, and TSC2 [42]. Less recurrent mutations detected in pancreatic NET involve ATM, YY1, and MUTYH [42, 45, 46]. TP53 and $R B 1$ are usually wild types in PanNET, in contrast to PanNEC $[15,16,47]$. A recent study focusing on the DNAmethylation profile distinguished between alpha-like, betalike, and intermediate PanNET clusters that differed in prognosis [48, 49].

Most NETs are sporadic and solitary tumors. However, approximately $10 \%$ of pancreatic NETs develop in association with genetic syndromes and then often manifest as multiple tumors, usually also affecting extrapancreatic organs. The most common syndrome is MEN 1 (see above), followed by the syndromes of Von-Hippel-Lindau, neurofibromatosis type 1 , and tuberous sclerosis, with germline mutations in the genes MEN1,VHL, NF1, and $T S C 2$, respectively. Functioning PanNETs occur predominantly in MEN1, in which they account for about $30 \%$ of the cases and include mainly insulinomas and duodenal gastrinomas. 


\section{PanNEC}

PanNECs are rare high-grade pancreatic neoplasms accounting for a maximum of $10 \%$ of PanNENs. They arise as sporadic, solitary, and non-functioning neoplasms, and have not been observed in association with genetic syndromes [1, 2]. An association with smoking can be suspected, has so far however not been established.

The histological profile of PanNECs is characterized by diffuse solid sheet-like and/or a more irregularly nested pattern (Fig. 3). Common to both patterns are geographical necrosis. NECs with diffuse sheet-like patterns are often composed of highly atypical small- to medium-sized cells that have a scant cytoplasm and hyperchromatic nuclei with inconspicuous nucleoli and focal nuclear molding. NECs with more nested patterns are mostly composed of larger cells whose cytoplasm is rather well-developed and eosinophilic, carrying a polymorphous nucleus with a prominent nucleolus within vesicular chromatin delimited by a delicate nuclear membrane. Mitoses, including atypical mitoses, are common. PanNECs are usually indistinguishable from NECs of other sites by histology alone $[1,2,4,16]$.

The immunohistochemical profile of PanNECs that is essential to establish the diagnosis includes the expression of cytokeratin (CK), synaptophysin, INSM1 (Fig. 3), chromogranin A, and Ki67, as well as the overexpression/loss of p53 (Fig. 3) and the loss of nuclear RB1 staining [1, 2, $15,16]$.

PanNECs express CK8 and 18. In small cell type PanNECs, CK labeling may show a punctuate pattern, and in exceptional cases, CK labeling can even be lacking. A few PanNECs also express vimentin. Synaptophysin is typically diffusely but faintly and somewhat patchy expressed, often displaying a dot-like pattern. Chromogranin A is usually focally and scarcely expressed and may even be lacking, since neurosecretory granules, whose membranes contain chromogranin A, are rare in NEC cells. CD56 labels the membranes of PanNECs broadly, but it should be never the only neuroendocrine marker on which the diagnosis of a NEC is based, since it has a high degree of unspecificity. PanNECs are mainly ISLET-1-negative [1-3, 8, 12, 15, 16, 50, 51]. All PanNECs show a Ki67 index greater than $20 \%$, with a mean of 50 to $60 \%$ (Table 1 ).

The molecular profile of PanNECs is characterized by TP53 and RB1 mutations which are the key drivers of PanNECs as well as of extrapancreatic NECs [4, 15, 16, 47, 52-54]. Later studies additionally identified $K R A S$ as a third driver in PanNEC [55], suggesting a potential relationship to PDAC. Next-generation sequencing studies using larger gene panels revealed no further recurrent gene mutations in PanNECs and no clear molecular differences between small and large cell subtypes [15]. However, NECs seem to possess an organ-specific signature, since PanNECs have only KRAS mutations, while colorectal NECs have also APC mutations $[15,52]$.

TP53 and RB1 mutations are important in the distinction of PanNEC from PanNET, as they are absent in G1/G2 PanNETs and are only occasionally present in PanNETs G3 [15, 16, 42]. Immunohistochemically, almost $70 \%$ of PanNECs overexpress p53 that reflects an underlying TP53 alteration, and show loss of RB1 nuclear staining, indicating a $R B 1$ alteration $[15,16]$. Unlike well-differentiated PanNETs, PanNECs retain the expression of DAXX/ATRX, since the corresponding genes are not mutated. SST2 expression is negative in $85 \%$ of the tumors [16]. These tumors, which are negative on somatostatin radio receptor scintigraphy, are often positive on FDG-PET [56].

\section{Mixed neuroendocrine-non-neuroendocrine neoplasms (MiNEN)}

PanNENs may contain coexisting high-grade PDAC or acinar cell carcinoma. If one component exceeds 30\% (an arbitrarily chosen threshold) of the total tumor cell population, such tumors are called "mixed neuroendocrine-non-neuroendocrine neoplasms (MiNENs)." If the non-neuroendocrine component is an adenocarcinoma and the neuroendocrine component presents as NEC, the old term "mixed adenoneuroendocrine neoplasm (MANEC)" can be retained [1, 2, 57]. In a small series of pancreatic MiNENs, the neuroendocrine as well as the non-neuroendocrine component displayed poor differentiation and either a mosaic or a composite/amphicrine pattern. Single cases of published pancreatic MiNEN revealed a close relationship to PDAC [15], as it was also found in colorectal MiNEN [52, 58], and interestingly also to its precursors, as two cases of pancreatic intraductal papillary mucinous neoplasms (IPMN) associated with NEN were reported, in which the NEN component showed GNAS mutations, typical for IPMN. In one case, the NEN component was a NEC [15], and in the other case, a NET [59]. In mixed acinar-neuroendocine carcinomas, the expression of trypsin and synaptophysin can be so intense and overlapping that an amphicrine pattern can be observed. Genetically and biologically, these neoplasms are closely related to the conventional acinar cell carcinomas $[60,61]$.

\section{Diagnostic pitfalls}

Pitfalls in the diagnosis of PanNENs concern mainly the confusion of NECs with NETs G3 and of NENs with a variety of non-NENs such as acinar cell carcinoma, solid pseudopapillary neoplasm, pancreatic paragangliomas, PDACs with neuroendocrine cells, and subsets of non-NENs of 
epithelial and mesenchymal origin with neuroendocrine features (Fig. 4) as well as tumor-like lesions [21, 62].

\section{PanNET G3 versus NEC}

The delimitation between NETs and NECs is important as their clinical management differs fundamentally [63]. In small biopsies and occasionally in resection specimens, the distinction between NETs G3 (also called high-grade NETs) and NECs, especially of large cell type, can be difficult.

Histologically, NETs G3 mostly show an organoid solid or solid-trabecular (rarely pseudoglandular) pattern that is well distinguishable from the usually sheet-like architecture of small cell NECs, but difficult to distinguish from the nested architecture of most large-cell NECs [15, 16, 64, 65], particularly in biopsies. Hyalinized versus desmoplastic stroma and regular versus random vessel pattern are also criteria for the distinction of NET from NEC, but are usually not helpful in biopsies. Helpful are cytological criteria, with more polymorphous nuclei with conspicuous nucleoli in NECs, and immunohistochemical markers, including SST2, p53, and RB1. The vast majority of NETs express SST2 and show a normal (weak and no more than 20\%) expression of p53 and RB1 (no complete loss) [16, 64, 65]. In contrast, only $16 \%$ of NEC express SST2 and show an abnormal expression of p53 and/or RB1 in 70\% of the cases [16]. If the distinction is still difficult, the testing for the expression of hormones can help, as most NETs, but no NECs, are hormone-producing tumors [10].

\section{Acinar cell carcinoma versus PanNET G3}

Acinar cell carcinomas are histologically recognized by their more or less striking acinar pattern, faint PAS positivity, round nuclei with prominent nucleoli, high mitotic rate, and scant fibrous stroma. However, some tumors displaying solid or trabecular patterns are very reminiscent of NETs [1]. In addition, approximately $40 \%$ of acinar cell carcinomas express synaptophysin and chromogranin $\mathrm{A}$, and some of these qualify as mixed acinar-neuroendocrine (ductal) carcinomas, with an intimate and amphicrine mixture of the two components. In the latter tumors, the co-expression of trypsin and synaptophysin can reach such an extent in all tumor cells that, if one only stains for synaptophysin, the tumor, which labels diffusely for synaptophysin, is easily misdiagnosed as NETs (especially NETs G3 when the mitotic activity is high) and only correctly recognized as mixed acinar-neuroendocrine carcinoma when trypsin (and/ or BCL10) is added to the marker panel (Fig. 5) [66-70]. Very helpful in these cases are SST2 and ISLET-1, since both markers are negative in acinar cell carcinomas with neuroendocrine features (authors' personal observation).

\section{Solid pseudopapillary neoplasm versus PanNET}

Solid pseudopapillary neoplasms may present with a predominantly solid and monomorphous cell pattern and the expression of synaptophysin and cytokeratin. These cases, which very much mimic a NET, however, differ from NETs by a lack of chromogranin A staining and a

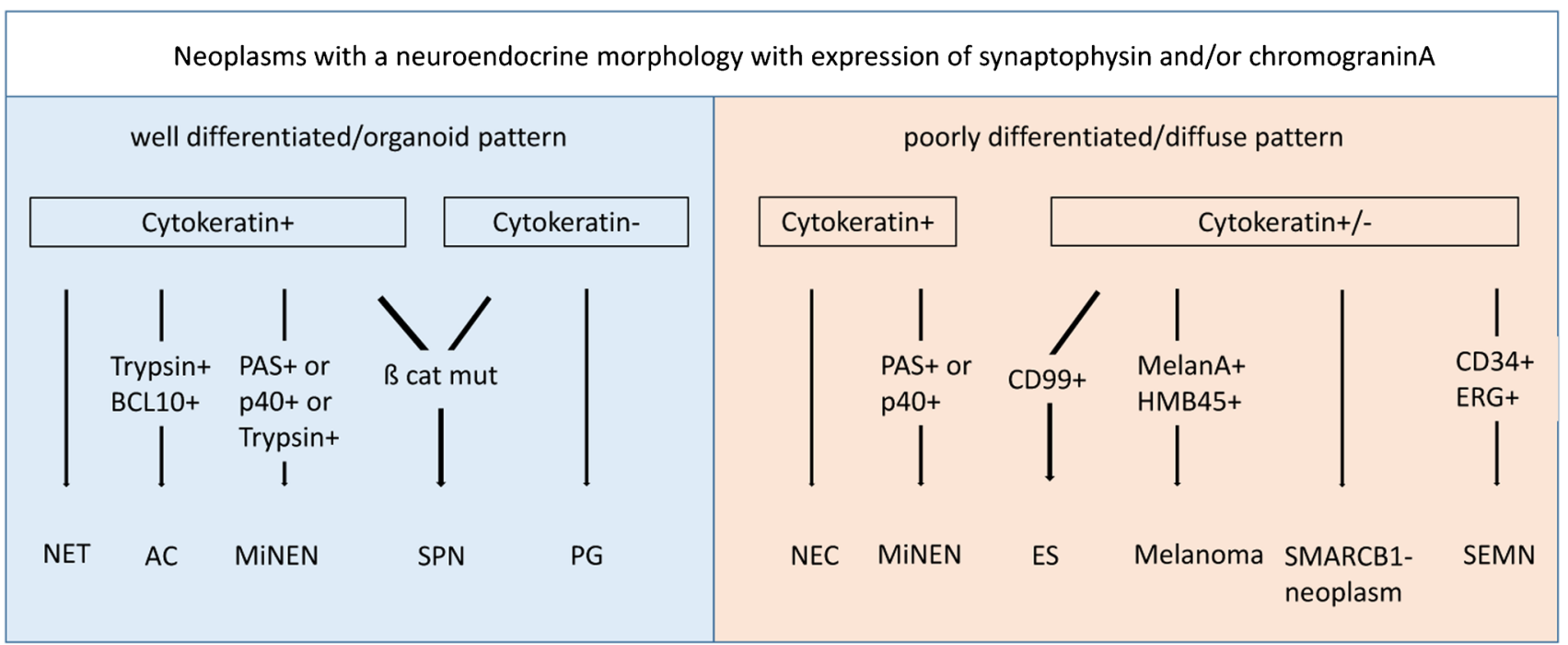

NET, neuroendocrine Tumor; AC, acinar cell carcinoma; MiNEN, mixed neuroendocrine-nonneuroendocrine neoplasm; SPN, solid pseudopapillary neoplasia; PG, paraganglioma; ES, Ewing sarcoma; SEMN, sclerosing epitheloid mesenchymal neoplasm

Fig. 4 Diagnostic algorithm for the differential diagnosis of synaptophysin expressing pancreatic neoplasms 
Fig. 5 Mixed acinar-neuroendocrine carcinoma with diffuse expression of synaptophysin (A) and trypsin (B)
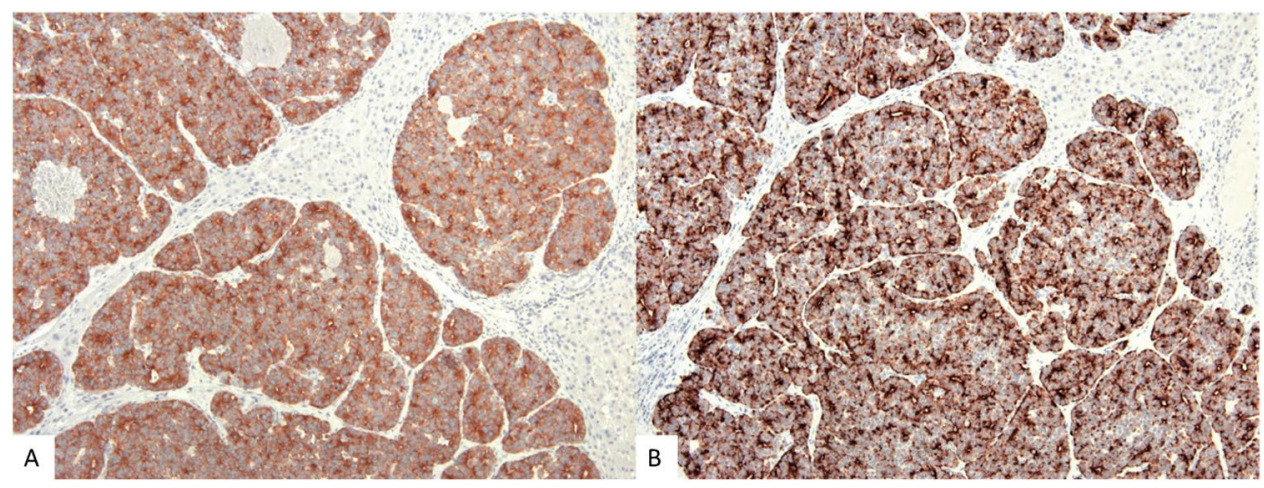

positive nuclear (and cytoplasmic) labeling for B-catenin, indicating a $C T N N B 1$-mutation found in almost all SPN [71-74]. Furthermore, it is helpful that the synaptophysin and cytokeratin staining in SPN is rarely diffuse, but usually patchy [62].

\section{Paraganglioma-like PanNET versus true paraganglioma}

A small fraction of PanNETs shows a solid, paragangliomalike histology and may therefore mimic rare paragangliomas occurring in or, more commonly, at the pancreas [10]. While paragangliomas usually show a benign behavior, paraganglioma-like PanNETs do not differ biologically from the other PanNETs. The key markers for the distinction of the two entities are CKs $[2,10]$ and GATA3 [75, 76]. GATA3 is positive in paragangliomas and negative in PanNETs, while CKs are negative in paragangliomas and positive in PanNETs. Furthermore, paragangliomas do not infiltrate into the pancreatic tissue, while paraganglioma-like PanNETs usually do $[10,23]$.

\section{Ductal adenocarcinoma with islet cells versus MiNEN}

The distinction of conventional PDAC intimately associated with islet cells from MiNEN is in most cases not a problem. First, the WHO classification requires that the neuroendocrine component of MiNENs exceeds $30 \%$ of the tumor cell population [1]. In PDAC, the number of islet cells that combine with duct-like glands of a PDAC to form ductalneuroendocrine complexes is less than $30 \%$ of the tumor cell population. In addition, they show no Ki67 labeling as a sign of proliferation in contrast to PDAC cells. Moreover, immunostains for pancreatic hormones identify the cells that associated with PDAC structures as islet cell types. Finally, the metastases of these PDACs never contain neuroendocrine cells of the type found in the pancreas [77].

\section{Mesenchymal and non-epithelial neoplasms versus NEC}

Recently a range of mesenchymal and non-epithelial mimickers of neuroendocrine neoplasms, mainly of the largecell NECs, have been described in a large consultation series [62]. Of particular interest and rather new among these NEN mimickers are tumors from the Ewing Sarcomas group, desmoplastic small round cell tumors, epithelioid neoplasms with FUS-CREM gene fusions, epithelioid sarcomas, synovial sarcomas, SMARCA4- and SMARCB1deficient neoplasms (Fig. 6), clear cell sarcomas of the gastrointestinal tract, alveolar soft part sarcomas, solitary fibrous tumors, chordomas, melanomas, and sclerosing epithelioid mesenchymal neoplasms. Six of these neoplasms were located in the pancreas and included Ewing sarcoma, SMARCB1(INI1)-deficient neoplasms (Fig. 6), melanoma, and sclerosing epithelioid sclerosing neoplasms [62]. To unmask these neoplasms as NEN mimickers, the testing of the key markers CD99, INI1, and S100 is necessary in cases of Ewing sarcomas, SMARCB1-deficient neoplasms, and melanomas, respectively. The sclerosing epithelioid mesenchymal neoplasm of the pancreas is an exceedingly rare pancreatic tumor that has recently been proposed as a new tumor entity with only single reported cases in the literature [78]. The synaptophysin expression in mesenchymal/ non-epithelial NEN mimickers is mostly patchy with coexpression of chromogranin $\mathrm{A}$ in one-third of the cases [62].

\section{Tumor-like lesions}

Tumor-like lesions as NEN mimickers are islet cell aggregates in specimens of chronic pancreatitis, particularly of the obstructive type of pancreatitis associated with duct occluding tumors. The islet clusters that are found in fibrotic and/ or lipomatous tissue devoid of acinar cells can histologically imitate an infiltrating PanNET. To avoid a diagnostic pitfall, the islet cell nature of the cell aggregates has to be demonstrated. This is easily done by immunostainings for insulin and glucagon, which reveal the normal non-random 
Fig. 6 Pancreatic SMARCB1 deficient rhabdoid neoplasm (A) with expression of synaptophy$\sin (\mathbf{B})$, chromogranin A (C), and expression loss of INI1 (D)
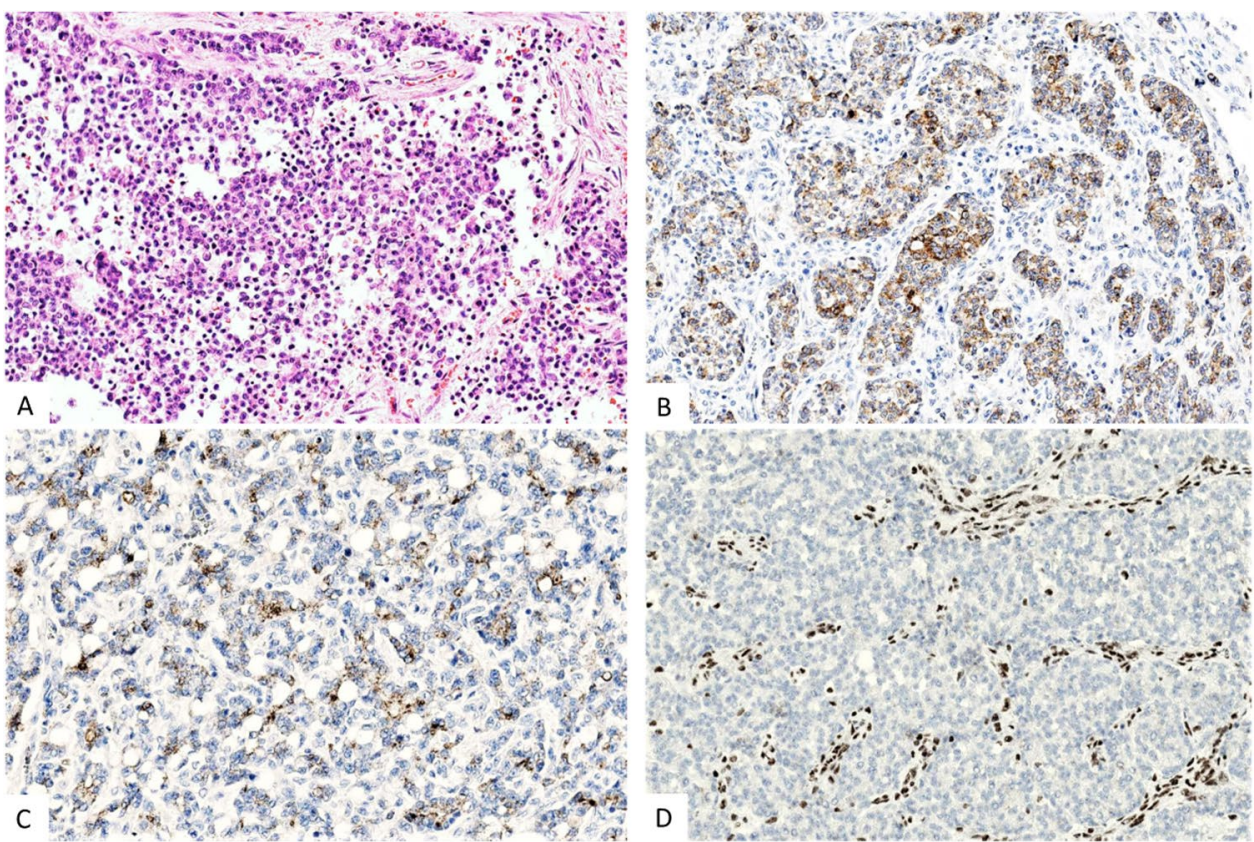

distribution of the two islet types in the islet cell clusters that profoundly differs from the monohormonal expression in most NETs [62]. The same applies to the PP-rich islet clusters in the posterior-caudal and uncinate lobe of the pancreatic head, when this part of the pancreas is resected with a PDAC. Again, these aggregates which can have a NET-like appearance reveal their normal islet cell type composition when tested for pancreatic polypeptide [79].

\section{Conclusion}

The diagnostic and therapeutic management of PanNENs has very much improved during the last two decades in which NET centers have emerged, supported by societies such as the European Neuroendocrine Tumor Society. This development was accompanied and supported by the steady improvement of classifications, diagnostic criteria, and prognostic assessments. The basis of all, the morphology, is still the starting point of diagnosis. The diagnostic criteria for assessing PanNEN histology have been refined and expanded at the same time in an attempt to capture the tumor's individuality, to which our attention is more and more directed. This path is continued by the use of biomarker immunohistology that reveals further properties of tumor cells which help to improve classification, precisely record proliferative activity, specify therapeutic approaches, and determine function. The final approach to the diagnosis is the genetic evaluation of the tumor which is currently increasingly integrated into our diagnostic pathways. The correct application of all the diagnostic criteria should protect us from misdiagnosis and pitfalls. Nevertheless, it is important to know and study the special pitfalls of NENs to avoid their potential confusion with NEN in general and PanNEN in particular.

Author contribution BK and GK: design of the manuscript; BK, MJ, $\mathrm{AK}$, and GK: drafting, reading, and revising the manuscript for important intellectual content and scientific integrity. All authors have read and approved the final manuscript.

Funding Open Access funding enabled and organized by Projekt DEAL.

\section{Declarations}

Ethical approval This work is compliant with ethical standards.

Conflict of interest The authors declare no competing interests.

Open Access This article is licensed under a Creative Commons Attribution 4.0 International License, which permits use, sharing, adaptation, distribution and reproduction in any medium or format, as long as you give appropriate credit to the original author(s) and the source, provide a link to the Creative Commons licence, and indicate if changes were made. The images or other third party material in this article are included in the article's Creative Commons licence, unless indicated otherwise in a credit line to the material. If material is not included in the article's Creative Commons licence and your intended use is not permitted by statutory regulation or exceeds the permitted use, you will need to obtain permission directly from the copyright holder. To view a copy of this licence, visit http://creativecommons.org/licenses/by/4.0/. 


\section{References}

1. Board WCoTE (2019) Digestive system tumours. 5th Edition ed. IARC, Lyon

2. Lloyd RV, Osamura RY, Klöppel G, Rosai J (2017) WHO classification of tumours of endocrine organs. IARC Press, Lyon

3. Klöppel G (2017) Neuroendocrine neoplasms: dichotomy, origin and classifications. Visc Med 33(5):324-330

4. Kasajima A, Klöppel G (2020) Neuroendocrine neoplasms of lung, pancreas and gut: a morphology-based comparison. Endocr Relat Cancer 27(11):R417-R432

5. Winter JM, Cameron JL, Campbell KA, Arnold MA, Chang DC, Coleman J, et al (2006) 1423 pancreaticoduodenectomies for pancreatic cancer: a single-institution experience. J Gastrointestin Surg 10(9):1199-210; discussion 210-1

6. El-Naggar AK, Chan JKC, Grandis JR, Takata T, Slootweg PJ (2017) WHO classification of head and neck tumours. IARC Press, Lyon

7. Travis WD, Brambilla E, Burke AP, Marx A, Nicholson AG (2015) WHO classification of tumours of the lung, pleura, thymus and heart. IARC Press, Lyon

8. Agaimy A, Erlenbach-Wunsch K, Konukiewitz B, Schmitt AM, Rieker RJ, Vieth M et al (2013) ISL1 expression is not restricted to pancreatic well-differentiated neuroendocrine neoplasms, but is also commonly found in well and poorly differentiated neuroendocrine neoplasms of extrapancreatic origin. Mod Pathol 26(7):995-1003

9. Konukiewitz B, Enosawa T, Klöppel G (2011) Glucagon expression in cystic pancreatic neuroendocrine neoplasms: an immunohistochemical analysis. Virchows Arch 458(1):47-53

10. Konukiewitz B, von Hornstein M, Jesinghaus M, Steiger K, Weichert W, Detlefsen S et al (2020) Pancreatic neuroendocrine tumors with somatostatin expression and paraganglioma-like features. Hum Pathol 102:79-87

11. Xue Y, Reid MD, Pehlivanoglu B, Obeng RC, Jiang H, Memis B et al (2020) Morphologic variants of pancreatic neuroendocrine tumors: clinicopathologic analysis and prognostic stratification. Endocr Pathol 31(3):239-253

12. Klöppel G (2011) Classification and pathology of gastroenteropancreatic neuroendocrine neoplasms. Endocr Relat Cancer 18(Suppl 1):S1-16

13. Kapran Y, Bauersfeld J, Anlauf M, Sipos B, Klöppel G (2006) Multihormonality and entrapment of islets in pancreatic endocrine tumors. Virchows Arch 448(4):394-398

14. Perren A, Couvelard A, Scoazec JY, Costa F, Borbath I, Delle Fave G et al (2017) ENETS consensus guidelines for the standards of care in neuroendocrine tumors: pathology: diagnosis and prognostic stratification. Neuroendocrinology 105(3):196-200

15. Konukiewitz B, Jesinghaus M, Steiger K, Schlitter AM, Kasajima A, Sipos B et al (2018) Pancreatic neuroendocrine carcinomas reveal a closer relationship to ductal adenocarcinomas than to neuroendocrine tumors G3. Hum Pathol 77:70-79

16. Konukiewitz B, Schlitter AM, Jesinghaus M, Pfister D, Steiger K, Segler A et al (2017) Somatostatin receptor expression related to TP53 and RB1 alterations in pancreatic and extrapancreatic neuroendocrine neoplasms with a Ki67-index above 20. Mod Pathol 30(4):587-598

17. Tanigawa M, Nakayama M, Taira T, Hattori S, Mihara Y, Kondo $\mathrm{R}$ et al (2018) Insulinoma-associated protein 1 (INSM1) is a useful marker for pancreatic neuroendocrine tumor. Med Mol Morphol 51(1):32-40

18. Bellizzi AM (2020) Immunohistochemistry in the diagnosis and classification of neuroendocrine neoplasms: what can brown do for you? Hum Pathol 96:8-33
19. Klöppel G, La Rosa S (2018) Ki67 labeling index: assessment and prognostic role in gastroenteropancreatic neuroendocrine neoplasms. Virchows Arch 472(3):341-349

20. Reid MD, Bagci P, Ohike N, Saka B, Erbarut Seven I, Dursun $\mathrm{N}$ et al (2015) Calculation of the Ki67 index in pancreatic neuroendocrine tumors: a comparative analysis of four counting methodologies. Mod Pathol 28(5):686-694

21. Kasajima A, Konukiewitz B, Schlitter AM, Weichert W, Klöppel G (2021) An analysis of 130 neuroendocrine tumors G3 regarding prevalence, origin, metastasis, and diagnostic features. Virchows Arch. https://doi.org/10.1007/s00428-021-03202-6

22. Krejs GJ, Orci L, Conlon JM, Ravazzola M, Davis GR, Raskin $\mathrm{P}$, et al (1979) Somatostatinoma syndrome. Biochemical, morphologic and clinical features. N Engl J Med 301(6):285-292

23. Garbrecht N, Anlauf M, Schmitt A, Henopp T, Sipos B, Raffel A et al (2008) Somatostatin-producing neuroendocrine tumors of the duodenum and pancreas: incidence, types, biological behavior, association with inherited syndromes, and functional activity. Endocr Relat Cancer 15(1):229-241

24. Uccella S, Blank A, Maragliano R, Sessa F, Perren A, La Rosa $S$ (2017) Calcitonin-producing neuroendocrine neoplasms of the pancreas: clinicopathological study of 25 cases and review of the literature. Endocr Pathol 28(4):351-361

25. Donow C, Pipeleers-Marichal M, Stamm B, Heitz PU, Klöppel G (1990) The pathology of insulinoma and gastrinoma. The location, size, multicentricity, association with multiple endocrine type-I neoplasms and malignancy. Dtsch Med Wochenschr 115(37):1386-1891

26. McCall CM, Shi C, Klein AP, Konukiewitz B, Edil BH, Ellison TA et al (2012) Serotonin expression in pancreatic neuroendocrine tumors correlates with a trabecular histologic pattern and large duct involvement. Hum Pathol 43(8):1169-1176

27. de Herder WW, Zandee WT, Hofland J (2000) Insulinoma. In: Feingold KR, Anawalt B, Boyce A, Chrousos G, de Herder WW, Dhatariya K, et al., editors. Endotext. South Dartmouth (MA)

28. Hermann G, Konukiewitz B, Schmitt A, Perren A, Klöppel G (2011) Hormonally defined pancreatic and duodenal neuroendocrine tumors differ in their transcription factor signatures: expression of ISL1, PDX1, NGN3, and CDX2. Virchows Arch 459(2): $147-154$

29. Jiao Y, Shi C, Edil BH, de Wilde RF, Klimstra DS, Maitra A et al (2011) DAXX/ATRX, MEN1, and mTOR pathway genes are frequently altered in pancreatic neuroendocrine tumors. Science 331(6021):1199-1203

30. Heaphy CM, de Wilde RF, Jiao Y, Klein AP, Edil BH, Shi C et al (2011) Altered telomeres in tumors with ATRX and DAXX mutations. Science 333(6041):425

31. de Wilde RF, Heaphy CM, Maitra A, Meeker AK, Edil BH, Wolfgang CL et al (2012) Loss of ATRX or DAXX expression and concomitant acquisition of the alternative lengthening of telomeres phenotype are late events in a small subset of MEN-1 syndrome pancreatic neuroendocrine tumors. Mod Pathol 25(7):1033-1039

32. Marinoni I, Kurrer AS, Vassella E, Dettmer M, Rudolph T, Banz $\mathrm{V}$, et al (2014) Loss of DAXX and ATRX are associated with chromosome instability and reduced survival of patients with pancreatic neuroendocrine tumors. Gastroenterology 146(2):453-60 e5

33. Di Domenico A, Wiedmer T, Marinoni I, Perren A (2017) Genetic and epigenetic drivers of neuroendocrine tumours (NET). Endocr Relat Cancer 24(9):R315-R334

34. Esposito I, Konukiewitz B, Schlitter AM, Klöppel G (2014) Pathology of pancreatic ductal adenocarcinoma: facts, challenges and future developments. World J Gastroenterol 20(38):13833-13841

35. Schlitter AM, Segler A, Steiger K, Michalski CW, Jager C, Konukiewitz B et al (2017) Molecular, morphological and 
survival analysis of 177 resected pancreatic ductal adenocarcinomas (PDACs): identification of prognostic subtypes. Sci Rep 7:41064

36. Esposito I, Segler A, Steiger K, Klöppel G (2015) Pathology, genetics and precursors of human and experimental pancreatic neoplasms: an update. Pancreatology 15(6):598-610

37. Larsson C, Skogseid B, Oberg K, Nakamura Y, Nordenskjold M (1988) Multiple endocrine neoplasia type 1 gene maps to chromosome 11 and is lost in insulinoma. Nature 332(6159):85-87

38. Agarwal SK, Guru SC, Heppner C, Erdos MR, Collins RM, Park SY et al (1999) Menin interacts with the AP1 transcription factor JunD and represses JunD-activated transcription. Cell 96(1):143-152

39. Dreijerink KMA, Timmers HTM, Brown M (2017) Twenty years of menin: emerging opportunities for restoration of transcriptional regulation in MEN1. Endocr Relat Cancer 24(10):T135-T145

40. Moore PS, Missiaglia E, Antonello D, Zamo A, Zamboni G, Corleto $\mathrm{V}$ et al (2001) Role of disease-causing genes in sporadic pancreatic endocrine tumors: MEN1 and VHL. Genes Chromosomes Cancer 32(2):177-181

41. Hackeng WM, Brosens LA, Poruk KE, Noe M, Hosoda W, Poling JS et al (2016) Aberrant Menin expression is an early event in pancreatic neuroendocrine tumorigenesis. Hum Pathol 56:93-100

42. Scarpa A, Chang DK, Nones K, Corbo V, Patch AM, Bailey P et al (2017) Whole-genome landscape of pancreatic neuroendocrine tumours. Nature 543(7643):65-71

43. Pipinikas CP, Dibra H, Karpathakis A, Feber A, Novelli M, Oukrif D et al (2015) Epigenetic dysregulation and poorer prognosis in DAXX-deficient pancreatic neuroendocrine tumours. Endocr Relat Cancer 22(3):L13-L18

44. Corbo V, Dalai I, Scardoni M, Barbi S, Beghelli S, Bersani S et al (2010) MEN1 in pancreatic endocrine tumors: analysis of gene and protein status in 169 sporadic neoplasms reveals alterations in the vast majority of cases. Endocr Relat Cancer 17(3):771-783

45. Cao Y, Gao Z, Li L, Jiang X, Shan A, Cai J et al (2013) Whole exome sequencing of insulinoma reveals recurrent T372R mutations in YY1. Nat Commun 4:2810

46. Corbo V, Beghelli S, Bersani S, Antonello D, Talamini G, Brunelli $M$ et al (2012) Pancreatic endocrine tumours: mutational and immunohistochemical survey of protein kinases reveals alterations in targetable kinases in cancer cell lines and rare primaries. Ann Oncol 23(1):127-134

47. Yachida S, Vakiani E, White CM, Zhong Y, Saunders T, Morgan $\mathrm{R}$ et al (2012) Small cell and large cell neuroendocrine carcinomas of the pancreas are genetically similar and distinct from well-differentiated pancreatic neuroendocrine tumors. Am J Surg Pathol 36(2):173-184

48. Di Domenico A, Pipinikas CP, Maire RS, Brautigam K, Simillion C, Dettmer MS et al (2020) Epigenetic landscape of pancreatic neuroendocrine tumours reveals distinct cells of origin and means of tumour progression. Commun Biol 3(1):740

49. Chan CS, Laddha SV, Lewis PW, Koletsky MS, Robzyk K, Da Silva E et al (2018) ATRX, DAXX or MEN1 mutant pancreatic neuroendocrine tumors are a distinct alpha-cell signature subgroup. Nat Commun 9(1):4158

50. Klöppel G (2013) Neuroendokrine Neoplasien. In: Stolte M, Rüschoff, J., Klöppel, G., editor. Pathologie - Verdauungstrakt und Peritoneum. Berlin Heidelberg

51. Klöppel G (2019) Neuroendocrine neoplasms : two families with distinct features unified in one classification (German version). Pathologe 40(3):211-219

52. Jesinghaus M, Konukiewitz B, Keller G, Kloor M, Steiger K, Reiche $\mathrm{M}$ et al (2017) Colorectal mixed adenoneuroendocrine carcinomas and neuroendocrine carcinomas are genetically closely related to colorectal adenocarcinomas. Mod Pathol 30(4):610-619
53. Kasajima A, Konukiewitz B, Oka N, Suzuki H, Sakurada A, Okada Y et al (2019) Clinicopathological profiling of lung carcinoids with a Ki67 index $>20$. Neuroendocrinology 108(2):109-120

54. Oka N, Kasajima A, Konukiewitz B, Sakurada A, Okada Y, Kameya T et al (2020) Classification and prognostic stratification of bronchopulmonary neuroendocrine neoplasms. Neuroendocrinology 110(5):393-403

55. Hijioka S, Hosoda W, Matsuo K, Ueno M, Furukawa M, Yoshitomi $\mathrm{H}$ et al (2017) Rb Loss and KRAS mutation are predictors of the response to platinum-based chemotherapy in pancreatic neuroendocrine neoplasm with grade 3: a Japanese multicenter pancreatic NEN-G3 Study. Clin Cancer Res 23(16):4625-4632

56. Ambrosini V, Kunikowska J, Baudin E, Bodei L, Bouvier C, Capdevila $\mathrm{J}$ et al (2021) Consensus on molecular imaging and theranostics in neuroendocrine neoplasms. Eur J Cancer 146:56-73

57. Rindi G, Arnold R, Bosman FT, Capella C, Klimstra DS, Klöppel $\mathrm{G}$, et al (2010) Nomenclature and classification of neuroendocrine neoplasms of the digestive system. In: Bosman FT, Carneiro F, Hruban RH, Theise ND, editors. WHO Classification of Tumours of the Digestive System. IARC: Lyon

58. Woischke C, Schaaf CW, Yang HM, Vieth M, Veits L, Geddert $\mathrm{H}$ et al (2017) In-depth mutational analyses of colorectal neuroendocrine carcinomas with adenoma or adenocarcinoma components. Mod Pathol 30(1):95-103

59. Schiavo Lena M, Cangi MG, Pecciarini L, Francaviglia I, Grassini G, Maire R et al (2021) Evidence of a common cell origin in a case of pancreatic mixed intraductal papillary mucinous neoplasm-neuroendocrine tumor. Virchows Arch 478(6):1215-1219

60. Bergmann F, Aulmann S, Sipos B, Kloor M, von Heydebreck A, Schweipert J et al (2014) Acinar cell carcinomas of the pancreas: a molecular analysis in a series of 57 cases. Virchows Arch 465(6):661-672

61. La Rosa S, Bernasconi B, Vanoli A, Sciarra A, Notohara K, Albarello L, et al (2018) c-MYC amplification and c-myc protein expression in pancreatic acinar cell carcinomas. New insights into the molecular signature of these rare cancers. Virchows Arch 473(4):435-441

62. Kasajima A, Konukiewitz B, Schlitter AM, Weichert W, Brasen JH, Agaimy A et al (2021) Mesenchymal/non-epithelial mimickers of neuroendocrine neoplasms with a focus on fusion geneassociated and SWI/SNF-deficient tumors. Virchows Arch. https:// doi.org/10.1007/s00428-021-03156-9

63. Garcia-Carbonero R, Sorbye H, Baudin E, Raymond E, Wiedenmann B, Niederle B et al (2016) ENETS consensus guidelines for high-grade gastroenteropancreatic neuroendocrine tumors and neuroendocrine carcinomas. Neuroendocrinology 103(2):186-194

64. Tang LH, Basturk O, Sue JJ, Klimstra DS (2016) A Practical Approach to the Classification of WHO Grade 3 (G3) Well-differentiated neuroendocrine tumor (WD-NET) and poorly differentiated neuroendocrine carcinoma (PD-NEC) of the pancreas. Am J Surg Pathol 40(9):1192-1202

65. Tang LH, Untch BR, Reidy DL, O'Reilly E, Dhall D, Jih L et al (2016) Well-differentiated neuroendocrine tumors with a morphologically apparent high-grade component: a pathway distinct from poorly differentiated neuroendocrine carcinomas. Clin Cancer Res 22(4):1011-1017

66. Hoorens A, Lemoine NR, McLellan E, Morohoshi T, Kamisawa T, Heitz PU, et al (1993) Pancreatic acinar cell carcinoma. An analysis of cell lineage markers, p53 expression, and Ki-ras mutation. Am J Pathol 143(3):685-698

67. Klimstra DS, Heffess CS, Oertel JE, Rosai J (1992) Acinar cell carcinoma of the pancreas. A clinicopathologic study of 28 cases. Am J Surg Pathol 16(9):815-837 
68. Ohike N, Kosmahl M, Klöppel G (2004) Mixed acinar-endocrine carcinoma of the pancreas. A clinicopathological study and comparison with acinar-cell carcinoma. Virchows Arch 445(3):231-235

69. Morohoshi T, Kanda M, Horie A, Chott A, Dreyer T, Klöppel G, et al (1987) Immunocytochemical markers of uncommon pancreatic tumors. Acinar cell carcinoma, pancreatoblastoma, and solid cystic (papillary-cystic) tumor. Cancer 59(4):739-747

70. La Rosa S, Franzi F, Marchet S, Finzi G, Clerici M, Vigetti D, et al (2009) The monoclonal anti-BCL10 antibody (clone 331.1) is a sensitive and specific marker of pancreatic acinar cell carcinoma and pancreatic metaplasia. Virchows Arch 454(2):133-142

71. Kosmahl M, Seada LS, Janig U, Harms D, Klöppel G (2000) Solid-pseudopapillary tumor of the pancreas: its origin revisited. Virchows Arch 436(5):473-480

72. La Rosa S, Bongiovanni M (2020) Pancreatic Solid pseudopapillary neoplasm: key pathologic and genetic features. Arch Pathol Lab Med 144(7):829-837

73. Papavramidis T, Papavramidis S (2005) Solid pseudopapillary tumors of the pancreas: review of 718 patients reported in English literature. J Am Coll Surg 200(6):965-972

74. Tanaka Y, Kato K, Notohara K, Hojo H, Ijiri R, Miyake T et al (2001) Frequent beta-catenin mutation and cytoplasmic/nuclear accumulation in pancreatic solid-pseudopapillary neoplasm. Cancer Res 61(23):8401-8404
75. Asa SL, Ezzat S, Mete O (2018) The diagnosis and clinical significance of paragangliomas in unusual locations. J Clin Med 7(9):280

76. Miettinen M, McCue PA, Sarlomo-Rikala M, Rys J, Czapiewski P, Wazny K et al (2014) GATA3: a multispecific but potentially useful marker in surgical pathology: a systematic analysis of 2500 epithelial and nonepithelial tumors. Am J Surg Pathol 38(1):13-22

77. Ohike N, Jurgensen A, Pipeleers-Marichal M, Klöppel G (2003) Mixed ductal-endocrine carcinomas of the pancreas and ductal adenocarcinomas with scattered endocrine cells: characterization of the endocrine cells. Virchows Arch 442(3):258-265

78. Basturk O, Weigelt B, Adsay V, Benhamida JK, Askan G, Wang L et al (2020) Sclerosing epithelioid mesenchymal neoplasm of the pancreas - a proposed new entity. Mod Pathol 33(3):456-467

79. Albers MB, Maurer E, Klöppel G, Bartsch DK (2014) Pancreatic polypeptide-rich islets in the posterior portion of the pancreatic head-a tumor mimic in somatostatin receptor scintigraphy. Pancreas 43(4):648-650

Publisher's note Springer Nature remains neutral with regard to jurisdictional claims in published maps and institutional affiliations. 